\title{
Risk of SARS-CoV-2 infection and subsequent hospital admission and death at different time intervals since first dose of COVID-19 vaccine administration, Italy, 27 December 2020 to mid-April 2021
} \author{
Italian Integrated Surveillance of COVID-19 study group $^{8}$, on behalf of the Italian COVID-19 vaccines registry ${ }^{9}$ \\ 1. Department of Infectious Diseases, Istituto Superiore di Sanità, Rome, Italy \\ (ECDC), Stockholm, Sweden \\ 3. National Centre for Drug Research and Evaluation, Istituto Superiore di Sanità, Rome, Italy \\ 4. Department of Informatics, Istituto Superiore di Sanità, Rome, Italy \\ 5. DG-SISS, Ministero della Salute, Rome, Italy \\ 6. Department of Neuroscience, Istituto Superiore di Sanità, Rome, Italy \\ 7. Research Coordination and Support Service, Istituto Superiore di Sanità, Rome, Italy \\ 8. The members have been listed at the end of this article \\ 9. The members have been listed at the end of this article
}

Alberto Mateo-Urdiales ${ }^{1,2}$, Stefania Spila Alegianiं ${ }^{3}$, Massimo Fabiani ${ }^{1}$, Patrizio Pezzotti ${ }^{1}$, Antonietta Filia ${ }^{1}$, Marco Massari ${ }^{3}$, Flavia Riccardo ${ }^{1}$, Marco Tallon', Valeria Proietti5, Martina Del Manso ${ }^{1,2}$, Maria Puopolo6, Matteo Spuri ${ }^{1}$, Cristina Morciano ${ }^{7}$, Fortunato (Paolo) D'Ancona ${ }^{1}$, Roberto Da Cas ${ }^{3}$, Serena Battilomo ${ }^{5}$, Antonino Bella ${ }^{1}$, Francesca Menniti-Ippolito ${ }^{3}$, on behalf of the

2. European Programme for Intervention Epidemiology Training (EPIET), European Centre for Disease Prevention and Control

Correspondence: Patrizio Pezzotti (patrizio.pezzotti@iss.it)

Mateo-Urdiales Alberto, Spila Alegiani Stefania, Fabiani Massimo, Pezzotti Patrizio, Filia Antonietta, Massari Marco, Riccardo Flavia, Tallon Marco, Proietti Valeria, Del Manso Martina, Puopolo Maria, Spuri Matteo, Morciano Cristina, D’Ancona Fortunato (Paolo), Da Cas Roberto, Battilomo Serena, Bella Antonino, MennitiIppolito Francesca, on behalf of the Italian Integrated Surveillance of COVID-19 study group, on behalf of the Italian COVID-19 vaccines registry. Risk of SARS-CoV-2 infection and subsequent hospital admission and death at different time intervals since first dose of COVID-19 vaccine administration, Italy, 27 December 2020 to mid-April 2021. Euro Surveill. 2021;26(25): pii=2100507. https://doi.org/10.2807/1560-7917.ES.2021.26.25.2100507

To assess the real-world impact of vaccines on COVID19 related outcomes, we analysed data from over 7 million recipients of at least one COVID-19 vaccine dose in Italy. Taking 0-14 days post-first dose as reference, the SARS-CoV-2 infection risk subsequently decreased, reaching a reduction by $78 \%$ (incidence rate ratios (IRR): $0.22 ; 95 \% \mathrm{Cl}: 0.21-0.24)$ 43-49 days post-first dose. Similarly, hospitalisation and death risks decreased, with 89\%(IRR:0.11; 95\% Cl:0.090.15 ) and $93 \%$ (IRR: $0.07 ; 95 \% \mathrm{Cl}: 0.04-0.11)$ reductions 36-42 days post-first dose. Our results support ongoing vaccination campaigns.

On 27 December 2020, Italy launched its vaccination campaign against coronavirus disease (COVID-19) subsequent to approval of the mRNA Comirnaty vaccine (BNT162b2 mRNA, BioNTech-Pfizer, Mainz, Germany/ New York, United States (US)). The mRNA Moderna COVID-19 Vaccine (mRNA-1273, Moderna, Cambridge, US) was approved on 14 January 2021, followed by the viral vector vaccine Vaxzevria (ChAdOx1 nCoV-19, Oxford-AstraZeneca, Cambridge, United Kingdom) on 2 February 2021 and the viral vector COVID-19 Vaccine Janssen (Ad26.COV2-S (recombinant), Janssen-Cilag International NV, Beerse, Belgium) on 12 March 2021 [1]. In the early stages, the campaign prioritised healthcare workers, residents in long-term care facilities
(LCFT) and persons aged over 80 years [2]. The purpose of this study is to assess risk of severe acute syndrome respiratory coronavirus 2 (SARS-CoV-2) infections, as well as related hospitalisations and deaths following COVID-19 vaccination in Italy.

\section{Retrieving data and measuring the impact of vaccination in Italy}

We conducted a cohort study among vaccinated individuals, aged 16 years and over, by linking COVID-19 vaccinated persons listed in the national vaccination registry of the Ministry of Health $(14,365,241$ on 3 May 2021), with data on positive cases of SARS-CoV-2 infection and related hospitalisations and deaths from the COVID-19 integrated surveillance system of the National Institute of Health, which we extracted on 2 May 2021 [3]. We excluded 567,162 (3.9\%) cases who were infected before being vaccinated and 76,573 (0.5\%) with missing information (Supplementary Material 1). Of the remaining $13,721,506$ persons, 8,389,595 (61\%) had received at least one dose of Comirnaty, 4,234,983 (31\%) of Vaxzevria, 1,021,134 (7\%) of Moderna and 75,794 (0.6\%) of Janssen. Individuals vaccinated with COVID-19 Vaccine Janssen were not included in the further analyses conducted in this study. Almost all study participants followed standard protocols of dose administration for each specific vaccine: $95 \%$ 
Timeline since the start of the vaccination campaign ${ }^{a}$ with the periods of selection and event ascertainment in the study population, Italy, 27 December 2020-2 May 2021

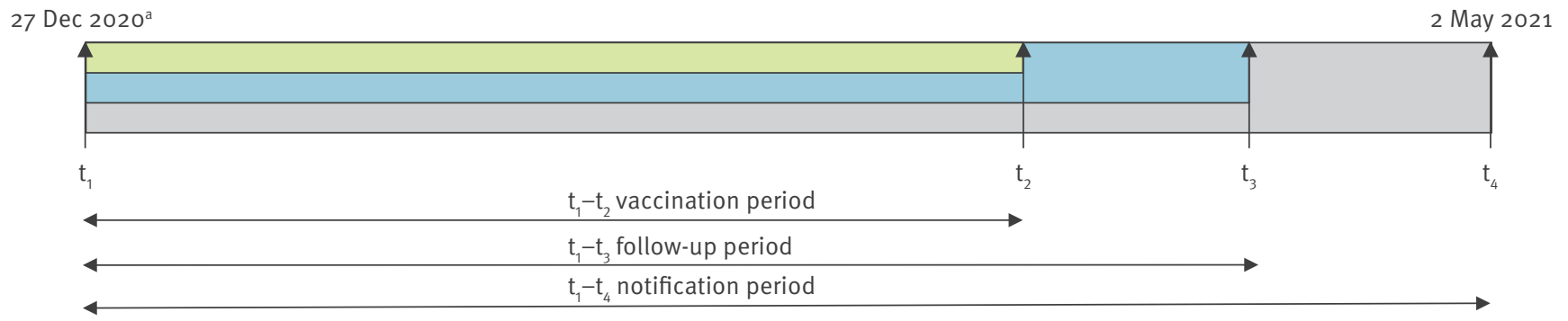

\begin{tabular}{|l|c|c|c|}
\hline \multirow{2}{*}{ Event } & \multicolumn{3}{|c|}{ Period } \\
\cline { 2 - 3 } & $\mathbf{t}_{1}-\mathbf{t}_{2}$ vaccination period & $\mathbf{t}_{1}-\mathbf{t}_{3}$ follow-up period & \multirow{2}{*}{$\mathbf{t}_{1}-\mathbf{t}_{4}$ notification period } \\
\hline Diagnosis of infection & 27 Dec 2020-4 Apr 2021 & 27 Dec 2020-18 Apr 2021 & \multirow{2}{*}{27 Dec 2020-2 May 2021 } \\
\hline Diagnosis of infection leading to hospitalisation & 27 Dec 2020-21 Mar 2021 & 27 Dec 2020-4 Apr 2021 \\
\hline Diagnosis of infection leading to death & 27 Dec 2020-7 Mar 2021 & 27 Dec 2020-21 Mar 2021 & \\
\hline
\end{tabular}

SARS-CoV-2: severe acute respiratory syndrome coronavirus 2; t: time.

a The vaccination campaign started on 27 December 2020.

$(5,049,606 / 5,298,025)$ received the second dose within 21-25 days from the first dose of Comirnaty, $95 \%(412,850 / 433,785)$ within $28-31$ days from the first dose of Moderna, and $95 \%(3,908 / 4,120)$ within 65-78 days from the first dose of Vaxzevria. In our analysis, 5,735,930 (42\%) participants received a second dose by 3 May. The follow-up time of each participant was measured from the date of the first dose administration up to the end of follow-up, independently of the possible administration of the second dose. We did not exclude persons who received the second dose of vaccine and we did not censor anyone at the second dose administration.

We analysed incidence rates of SARS-CoV-2 infection in 7,370,008 persons who had received a first dose of vaccine by 4 April 2021. Data were extracted on 2 May 2021 , to allow for at least 2 weeks of follow-up after vaccination and another 2 weeks of possible delays in notification of the infection to the surveillance system. To measure hospitalisation rates, we included persons who had received a first dose of vaccine by 21 March $2021(n=5,133,899)$, to allow for a longer observation period, considering that a possible worsening of symptoms and hospitalisation may occur sometime after diagnosis, and for time to report the occurrence of hospital admission to the surveillance system. For the same reasons, we measured death rates by including all persons vaccinated by 7 March $2021(n=3,622,029)$ (Figure 1).

To measure the impact of vaccination, we calculated rates (as ratios of numbers of events to person-days of follow-up) of infection, hospitalisation and death in two periods: days $0-14$ post-first dose and from 15 days after the first dose until the end of follow-up. In line with current scientific evidence, we considered rates in the first 14 days after the first dose as reference, assuming that during this period the risk of acquiring the infection is comparable to the unvaccinated population [4-6]. We then calculated incidence, hospitalisation and death rates by 7 -day time-intervals until the end of follow-up. Hospitalisations and deaths were assigned to the dates when diagnosis of infection with SARS-CoV-2 occurred, and not to the period in which these events took place, given that we aimed to measure rates of infections leading to hospitalisation/death, not the time when these events per se took place.

Finally, we carried out a multivariable analysis on individual data split by period (i.e. 0-14 days and subsequent weekly time-intervals from the first dose). We used Poisson regression models, with robust variance estimation, to calculate incidence rate ratios (IRRS) with $95 \%$ confidence intervals $(\mathrm{Cl})$ measuring changes in the risk of infection, hospitalisation and death over different time periods after vaccination, compared with the initial 14-day period. To consider only events associated with COVID-19, we adopted a conservative approach including only hospitalisations and deaths occurring within 30 days from diagnosis of SARSCoV-2 infection, which amount, respectively, to $99 \%$ $(366,921 / 372,268)$ and $89 \%(107,671 / 121,075)$ of all those registered in the surveillance database who were hospitalised or died from the start of the epidemic in Italy (20 February 2020) until 2 May 2021. We included sex, age group, region of vaccination, vaccine brand, and vaccination priority group as covariates. Estimates were also adjusted for calendar week of administration of first vaccine dose and for regional weekly incidence 
Rates of SARS-CoV-2 infection, related hospitalisation and death 0-14 days after administration of first COVID-19 vaccine dose and thereafter, Italy, 27 December 2020-18 April 2021

\begin{tabular}{|c|c|c|c|c|c|c|c|c|c|}
\hline \multirow{3}{*}{\multicolumn{2}{|c|}{$\begin{array}{l}\text { Characteristics of persons involved in } \\
\text { the study }\end{array}$}} & \multirow[b]{3}{*}{$\begin{array}{c}\text { Total } \\
\text { vaccinated }\end{array}$} & \multicolumn{6}{|c|}{ Period from administration of first dose } & \multirow[b]{3}{*}{$p$ value } \\
\hline & & & \multicolumn{3}{|c|}{$0-14$ days } & \multicolumn{3}{|c|}{$>14$ days } & \\
\hline & & & Person-days & Events & $\begin{array}{c}\text { Rate } \\
\text { (per 10,000 } \\
\text { person-days) }\end{array}$ & Person-days & Events & \begin{tabular}{|c|} 
Rate \\
(per 10,000 \\
person-days)
\end{tabular} & \\
\hline \multicolumn{10}{|l|}{ SARS-CoV-2 infection } \\
\hline \multicolumn{2}{|l|}{ Total } & $7,370,008$ & $102,991,407$ & 29,839 & 2.90 & $240,605,533$ & 32,020 & 1.33 & $<0.001$ \\
\hline \multirow{4}{*}{ Age group in years } & $<40$ & $1,098,052$ & $15,334,867$ & 5,605 & 3.66 & $48,764,109$ & 6,143 & 1.26 & $<0.001$ \\
\hline & $\begin{array}{c}\text { (per } 10,000 \\
\text { person-days) }\end{array}$ & $2,042,446$ & $28,529,032$ & 10,102 & 3.54 & $85,752,132$ & 11,962 & 1.39 & $<0.001$ \\
\hline & $60-79$ & $1,714,155$ & $23,966,168$ & 5,141 & 2.15 & $41,622,567$ & 4,479 & 1.08 & $<0.001$ \\
\hline & $\geq 80$ & $2,515,355$ & $35,161,340$ & 8,991 & 2.56 & $64,466,725$ & 9,436 & 1.46 & $<0.001$ \\
\hline \multirow{2}{*}{ Sex } & Males & $3,055,506$ & $42,700,832$ & 12,039 & 2.82 & $95,747,599$ & 11,835 & 1.24 & 0.001 \\
\hline & Females & $4,314,502$ & $60,290,577$ & 17,800 & 2.95 & $144,857,934$ & 20,185 & 1.39 & $<0.001$ \\
\hline \multirow{7}{*}{$\begin{array}{l}\text { Date of immunisation } \\
\text { with a first dose of } \\
\text { COVID-19 vaccine }\end{array}$} & 27 Dec-15 Jan & $1,011,356$ & $14,100,876$ & 8,555 & 6.07 & $85,029,552$ & 11,088 & 1.30 & $<0.001$ \\
\hline & $16 \operatorname{Jan}-30 \operatorname{Jan}$ & 229,767 & $3,206,796$ & 1,484 & 4.63 & $16,973,189$ & 2,177 & 1.28 & $<0.001$ \\
\hline & $31 \mathrm{Jan}-14 \mathrm{Feb}$ & 340,052 & $4,753,120$ & 1,264 & 2.66 & $17,962,690$ & 2,086 & 1.16 & $<0.001$ \\
\hline & $15 \mathrm{Feb}-1 \mathrm{Mar}$ & $1,275,340$ & $17,827,593$ & 4,625 & 2.59 & $50,920,224$ & 6,654 & 1.31 & $<0.001$ \\
\hline & 2 Mar-16 Mar & $1,831,139$ & $25,592,249$ & 7,098 & 2.77 & $48,233,854$ & 7,228 & 1.50 & $<0.001$ \\
\hline & 17 Mar-28 Mar & $1,512,076$ & $21,142,756$ & 4,223 & 2.00 & $17,657,048$ & 2,288 & 1.30 & $<0.001$ \\
\hline & $29 \mathrm{Mar}-4 \mathrm{Apr}$ & $1,170,278$ & $16,368,017$ & 2,590 & 1.58 & $3,828,976$ & 499 & 1.30 & $<0.001$ \\
\hline \multicolumn{10}{|c|}{ COVID-19 hospitalisation } \\
\hline \multicolumn{2}{|l|}{ Total } & $5,133,899$ & $71,852,063$ & 3,175 & 0.44 & $155,833,205$ & 1,797 & 0.12 & $<0.001$ \\
\hline \multirow{4}{*}{ Age group in years } & $<40$ & 890,565 & $12,466,598$ & 93 & 0.07 & $35,107,107$ & 33 & 0.01 & $<0.001$ \\
\hline & $40-59$ & $1,643,782$ & $23,009,741$ & 353 & 0.15 & $60,464,621$ & 144 & 0.02 & $<0.001$ \\
\hline & $60-79$ & 812,377 & $11,369,440$ & 525 & 0.46 & $25,025,547$ & 239 & 0.10 & $<0.001$ \\
\hline & $\geq 80$ & $1,787,175$ & $25,006,284$ & 2,204 & 0.88 & $35,235,931$ & 1,381 & 0.39 & $<0.001$ \\
\hline \multirow{2}{*}{ Sex } & Males & $2,077,697$ & $29,076,046$ & 1,688 & 0.58 & $60,964,774$ & 835 & 0.14 & $<0.001$ \\
\hline & Females & $3,056,202$ & $42,776,017$ & 1,487 & 0.35 & $94,868,431$ & 962 & 0.10 & $<0.001$ \\
\hline \multirow{6}{*}{$\begin{array}{l}\text { Date of immunisation } \\
\text { with a first dose of } \\
\text { COVID-19 vaccine }\end{array}$} & $27 \mathrm{Dec}-15 \mathrm{Jan}$ & $1,069,755$ & $14,153,395$ & 585 & 0.41 & $71,531,426$ & 423 & 0.06 & $<0.001$ \\
\hline & $16 \operatorname{Jan}-30 \operatorname{Jan}$ & 171,252 & $3,215,357$ & 152 & 0.47 & $13,874,842$ & 123 & 0.09 & $<0.001$ \\
\hline & $31 \mathrm{Jan}-14$ Feb & 314,180 & $4,759,689$ & 99 & 0.21 & $13,309,313$ & 100 & 0.08 & $<0.001$ \\
\hline & 15 Feb-1 Mar & $1,126,326$ & $17,849,208$ & 759 & 0.43 & $33,370,730$ & 552 & 0.17 & $<0.001$ \\
\hline & 2 Mar-16 Mar & $2,005,929$ & $25,626,251$ & 1,245 & 0.49 & $22,882,081$ & 558 & 0.24 & $<0.001$ \\
\hline & 17 Mar-28 Mar & 446,457 & $6,248,165$ & 335 & 0.54 & 864,813 & 41 & 0.47 & 0.464 \\
\hline \multicolumn{10}{|l|}{ COVID-19 death } \\
\hline \multicolumn{2}{|l|}{ Total } & $3,622,029$ & $50,701,430$ & 899 & 0.18 & $94,439,262$ & 418 & 0.04 & $<0.001$ \\
\hline \multirow{4}{*}{ Age group in years } & $<40$ & 714,934 & $10,008,516$ & 0 & 0.00 & $23,815,086$ & 0 & 0.00 & NC \\
\hline & $40-59$ & $1,270,264$ & $17,783,149$ & 7 & 0.00 & $39,777,616$ & 8 & 0.00 & 0.207 \\
\hline & $60-79$ & 533,419 & $7,466,957$ & 111 & 0.15 & $15,939,429$ & 38 & 0.02 & $<0.001$ \\
\hline & $\geq 80$ & $1,103,412$ & $15,442,809$ & 781 & 0.51 & $14,907,130$ & 372 & 0.25 & $<0.001$ \\
\hline \multirow{2}{*}{ Sex } & Males & $1,437,537$ & $20,122,280$ & 443 & 0.22 & $36,295,566$ & 167 & 0.05 & $<0.001$ \\
\hline & Females & $2,184,492$ & $30,579,151$ & 456 & 0.15 & $58,143,696$ & 251 & 0.04 & $<0.001$ \\
\hline \multirow{5}{*}{$\begin{array}{l}\text { Date of immunisation } \\
\text { with a first dose of } \\
\text { COVID-19 vaccine }\end{array}$} & 27 Dec-15 Jan & $1,069,850$ & $14,156,286$ & 360 & 0.25 & $57,579,446$ & 192 & 0.03 & $<0.001$ \\
\hline & $16 \operatorname{Jan}-30$ Jan & 171,272 & $3,216,218$ & 78 & 0.24 & $10,696,048$ & 53 & 0.05 & $<0.001$ \\
\hline & $31 \mathrm{Jan}-14 \mathrm{Feb}$ & 314,195 & $4,760,332$ & 42 & 0.09 & $8,571,541$ & 32 & 0.04 & $<0.001$ \\
\hline & 15 Feb-1 Mar & $1,126,356$ & $17,852,787$ & 260 & 0.15 & $15,555,615$ & 117 & 0.08 & $<0.001$ \\
\hline & 2 Mar-16 Mar & 940,356 & $10,715,807$ & 159 & 0.15 & $2,036,612$ & 24 & 0.12 & 0.293 \\
\hline
\end{tabular}

COVID-19: coronavirus disease; SARS-CoV-2: severe acute respiratory syndrome coronavirus 2; NC: not calculable.

${ }^{a} M$ id $\mathrm{p}$ values adjusted test of incidence-rate difference.

Reduction in the adjusted risk of infection, hospitalisation and death in vaccinated persons by 7 -days with respect to the first 14 days postfirst COVID-19 vaccine dose. 


\section{FIGURE 2}

Adjusted incidence rate ratios with 95\% confidence intervals of SARS-CoV-2 (A) infection, (B) hospitalisation and (C) death, by 7 -day period with respect to the first 14 days post first-dose of COVID-19 vaccine, Italy, 27 December 2020-18 April 2021

A. Infection

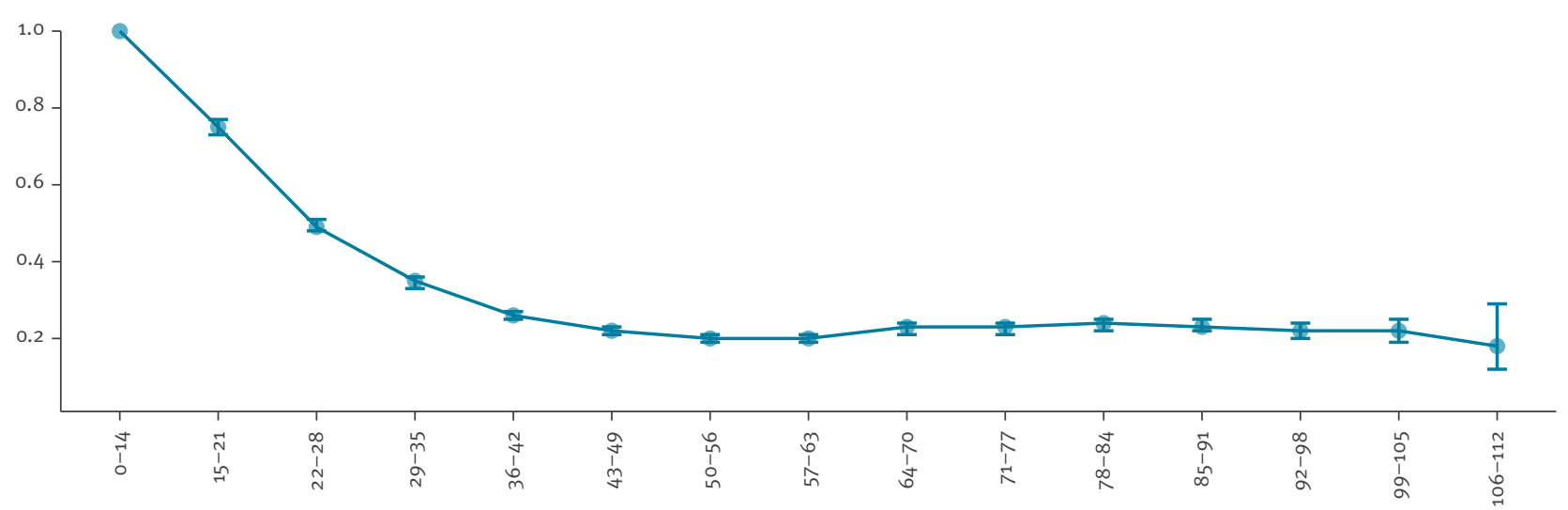

B. Hospitalisation

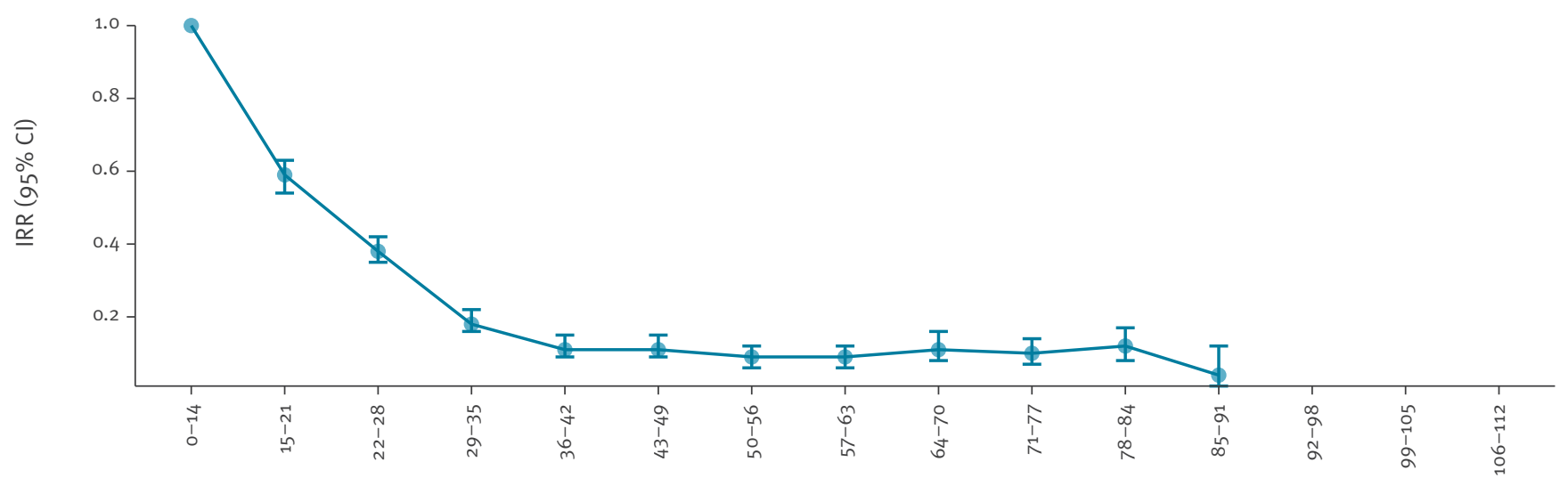

C. Death

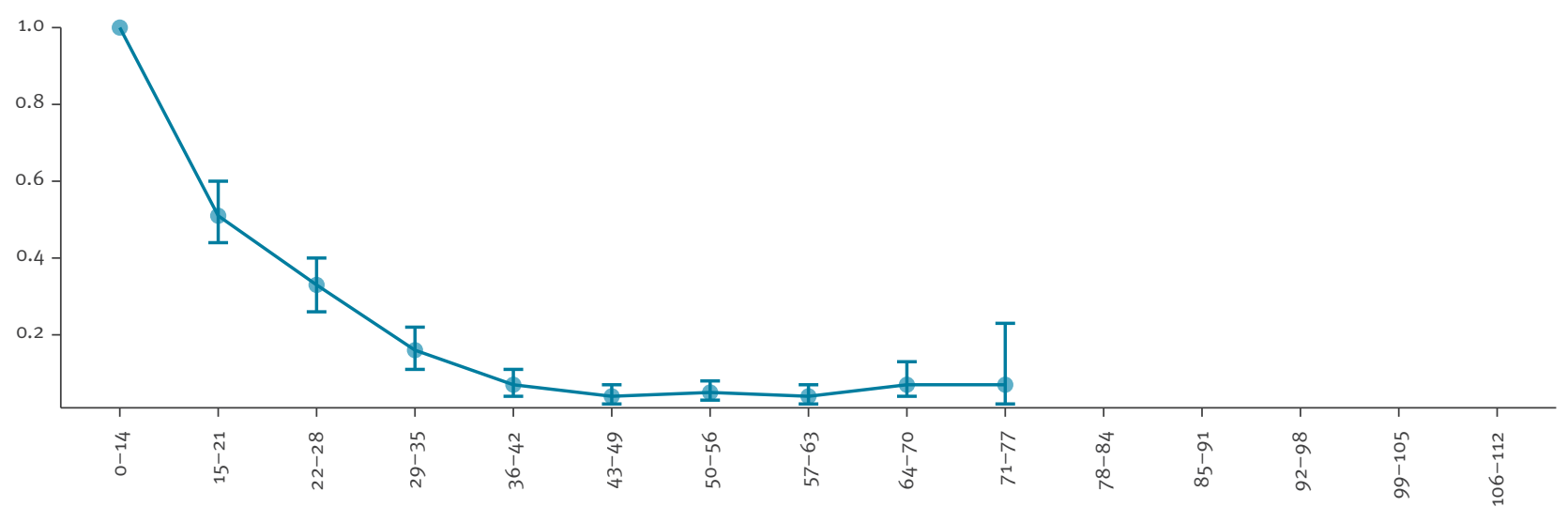

Period interval from first dose (days)

$\mathrm{Cl}$ : confidence interval; COVID-19: coronavirus disease; IRR: incidence rate ratio; SARS-CoV-2: severe acute respiratory syndrome coronavirus 2. 
in the general population to consider time-related and geographical variations in the probability of infection.

\section{Ethical statement}

The dissemination of COVID-19 surveillance data was authorised by the Italian Presidency of the Council of Ministers on 27 February 2020 (Ordinance no. 640).

\section{SARS-CoV-2 infection, related hospitalisation and death rates in the first 14 days after first COVID-19 vaccine dose and afterwards}

Compared with the reference period, we observed a reduction in the overall incidence of infection (2.9 vs 1.3 per 10,000 person-days), hospitalisation (0.44 vs 0.12 ) and death (0.18 vs 0.04 ), both overall and stratifying by age group, sex, geographical location and calendar period (Table).

The adjusted risk of SARS-CoV-2 infection decreased gradually until the period 42-49 days post-first dose, with a reduction of up to $78 \%$ (IRR: 0.22 ; $95 \% \mathrm{Cl}: 0.21-$ 0.24). Subsequently, the risk level stabilised. Similarly, the risk of hospitalisation and death among reported COVID-19 cases decreased gradually until the period 35-42 days post-first dose, when the estimated risk reduction was 89\% (IRR:0.11;95\% Cl:0.09-0.15) for hospitalisation and 93\% (IRR: $0.07 ; 95 \% \mathrm{Cl}: 0.04-0.11$ ) for death. After this period, the risks remained stable (Figure 2).

The analyses by age and priority group confirm a gradual decrease in the risk of infection from 14 to 42 days after first dose with an approximate risk reduction of $80 \%$ compared with the first 14 days post-first dose. Towards the end of the follow-up period (99-105 days post-first dose), the risk reduction was significantly more pronounced in residents in LCTF (IRR: $0.06 ; 95 \% \mathrm{Cl}: 0.04-0.11$ ) than in HCWs (IRR:0.21;95\% Cl:0.18-0.24). Equally, during the same period, the risk reduction in those aged over 80 years of age (IRR:0.08;95\% Cl:0.04-0.14) was significantly higher than in those aged 40-59years (IRR: $0.26 ; 95 \% \mathrm{Cl}: 0.22-0.31$ ) and aged 40 years or less (IRR: $0.24 ; 95 \% \mathrm{Cl}: 0.19-0.30$ ) (Figure 3).

\section{Discussion}

Our results suggest a significantly reduced risk of SARS-CoV-2 infection, and COVID-19 related hospitalisation and death in vaccinated individuals from 2 weeks post-vaccination with a first dose compared with the period comprising the first 2 weeks following vaccination. Risk reduction gradually increased from week 2 post-first dose until weeks 5-6, after which it remained stable.

We analysed over 7 million vaccinated people, the largest cohort used to date, to assess the effect of COVID19 vaccines. This allowed us to adjust the estimates for several possible confounding factors and to stratify by age and priority groups.
Results agree with previous reports in finding COVID19 vaccines to be effective in preventing SARS-CoV-2 infection. Four studies focused on the Comirnaty vaccine reported risk reduction estimates of $85-95 \%$ 1 week after second dose [7-10] and a study including both Comirnaty and Moderna found a $94 \%$ risk reduction in persons aged 65 years and older [11]. With regards to hospitalisations, risk reductions of up to $87 \%$ with Comirnaty and $84 \%$ with Comirnaty and Vaxzevria have been reported, which are similar to our $89 \%$ estimate $[7,12]$. Finally, evidence from Israel suggest that Comirnaty provides, from 1 week after the second dose, a $96.7 \%$ reduction of the risk of dying, similar to our estimate of $93 \%$ [9].

Unlike previous studies [9], we found higher risk reductions in older age groups than in the younger population, and in persons living in LCTF compared with HCW. These findings could have several explanations. As residents and staff of LCTF were among the first to be vaccinated, it is possible that residents in these facilities may have benefited from some degree of community protection, especially in a context of strict control of outside visitors. It is also possible, however, that estimates in this population, and in the older age groups, are biased towards overestimation of the risk reduction due to lack of survival ascertainment. As we only record deaths in SARS-CoV-2 positive individuals, persons dying from other causes - and therefore no longer at risk of infection - are still considered alive and therefore at risk.

Prior published studies used a control group of unvaccinated persons to estimate risk reduction, whereas we used the first 14 days post-first dose as the reference period. This approach has its strengths and limitations. Although studies have found that COVID-19 vaccines only start to provide significant protection after 2 weeks post-first dose [4-6], the immune response triggered by vaccination is gradual, and could already confer some degree of protection in the last days of this period. In this sense, for example, a study found a lower risk of infection in days 10-13 post-first COVID19 vaccine dose compared with the previous period, though not significantly lower than in the unvaccinated population [8]. It is possible that our estimates of risk reduction are partially underestimating the protective effect of vaccines. As this study did not collect data on the unvaccinated population, it was not possible to conduct a sensitivity analysis with unvaccinated people as a control. On the other hand, being vaccinated in the first stages of the vaccination campaign could be influenced by strategical (e.g. high-risk groups), behavioural (e.g. vaccine hesitancy) and/or psychological (e.g. self-perceived risk) factors which could differ systematically between the vaccinated and the unvaccinated populations. These factors, if not accounted for, could introduce biases that might be avoided by using the same population as the reference group. To test the robustness of our results, we carried out a sensitivity 


\section{FIGURE 3}

Adjusted incidence rate ratios with 95\% confidence intervals of SARS-CoV-2 infection in (A) healthcare workers, (B) residents of long-term care facilities and (C-F) age groups, by 7-day period with respect to the first 14 days post first-dose of COVID-19 vaccine, Italy, 27 December 2020-18 April 2021

A. HCW

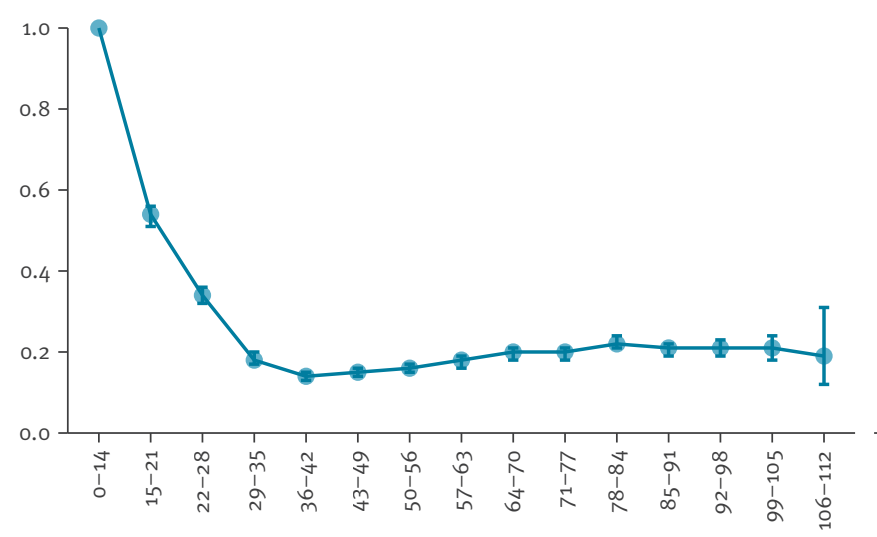

C. $<40$ years old

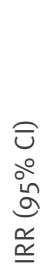

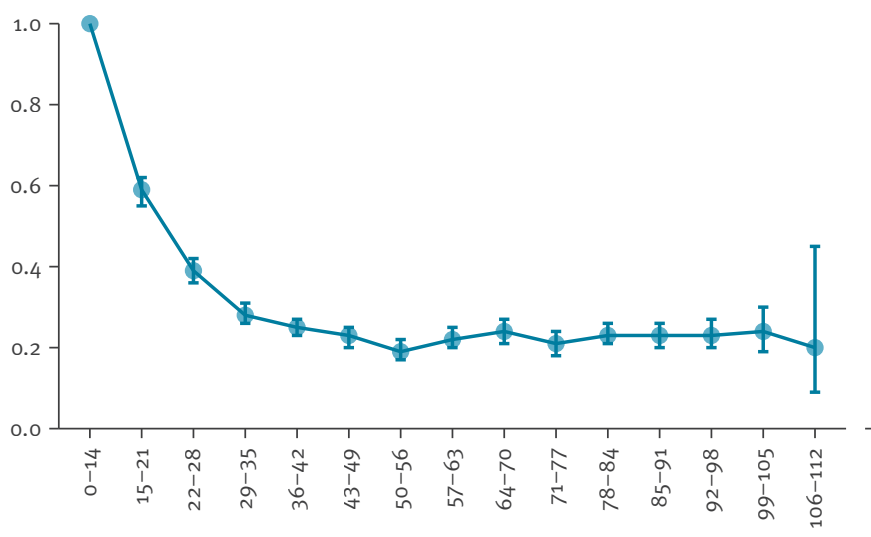

B. LCTF

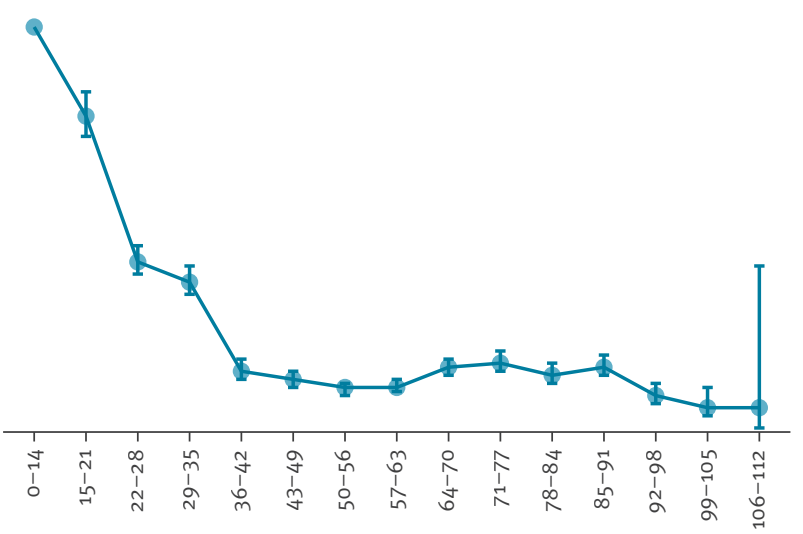

D. 40-59 years old

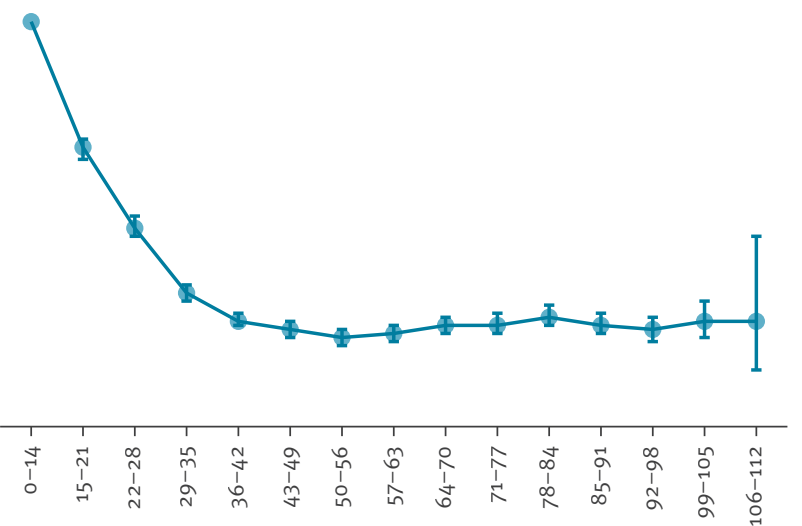

F. $\geq 80$ years old
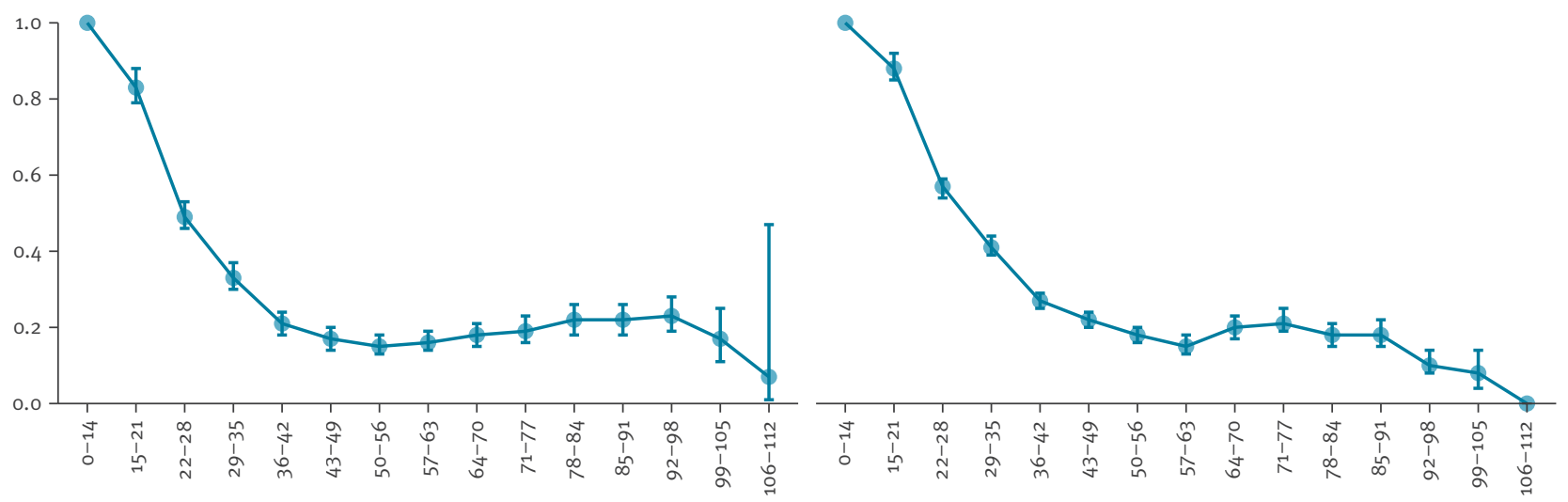

Period interval from first dose (days)

$\mathrm{Cl}$ : confidence interval; COVID-19: coronavirus disease; HCW: healthcare worker; IRR: incidence risk ratios (adjusted); LTCF: long-term care facility residents, SARS-CoV-2: severe acute respiratory syndrome coronavirus 2. 
analysis using the first 7 days post-first dose as the reference period. We found that during the period 7-14 days post-first dose, the risk of hospitalisation and death was similar to the reference period, but that the risk of infection was higher (Supplementary Material 2). One explanation for this finding is that vaccinated individuals may be less likely to respect social-distancing rules in the immediate days after vaccination [13]. As immunity is not yet complete during this period, this leads to a higher-than-expected infection rate. However, estimates of the risk reduction in the weekly time intervals from 14 days post-vaccination onwards were very close to those obtained using the whole period 0-14 days post vaccination as reference.

Furthermore, other factors could have affected the precision of our estimates. For example, it is possible that vaccinated individuals may be less likely to seek testing for SARS-CoV-2 infection if asymptomatic or only mildly symptomatic. As we only have information on cases notified through the surveillance system, this could also lead to an overestimation of our risk reduction estimates. Finally, we did not compare different brands or vaccines types (mRNA, viral vector), since different vaccines were introduced in different time periods, have different time schedules and were administered to population groups with differing risk profiles. A longer follow-up time is required for meaningful comparisons.

\section{Conclusion}

Compared with the first 2 weeks after the first dose, the risk of infection, hospitalisation and death from SARS-CoV-2 decreases gradually and substantially in vaccinated individuals. These results suggest that the vaccination campaign has the potential to significantly reduce the burden of COVID-19 in terms of morbidity and mortality if high levels of vaccine uptake are achieved.

\section{Italian Integrated Surveillance of COVID-19 study group}

ISS coordination group: Antonino Bella, Martina Del Manso, Xanthi Andrianou, Alberto Mateo Urdiales, Massimo Fabiani, Matteo Spuri, Stefania Bellino, Stefano Boros, Maria Cristina Rota, Ornella Punzo, Maria Fenicia Vescio, Daniele Petrone, Marco Tallon, Corrado Di Benedetto, Alessandra Ciervo, Paola Stefanelli, Flavia Riccardo, Patrizio Pezzotti.

Regional representatives: Antonia Petrucci (Abruzzo); Michele La Bianca (Basilicata); Anna Domenica Mignuoli (Calabria); Pietro Buono (Campania); Erika Massimiliani (Emilia-Romagna); Fabio Barbone (Friuli Venezia Giulia); Francesco Vairo (Lazio); Camilla Sticchi (Liguria); Danilo Cereda (Lombardia); Lucia Di Furia (Marche); Francesco Sforza (Molise); Annamaria Bassot (P.A. Bolzano); Pier Paolo Benetollo (P.A. Trento); Chiara Pasqualini (Piemonte); Lucia Bisceglia (Puglia); Maria Antonietta Palmas (Sardegna); Salvatore Scondotto (Sicilia); Emanuela Balocchini (Toscana); Anna Tosti (Umbria); Mauro Ruffier (Valle D'Aosta); Filippo Da Re (Veneto).
Italian COVID-19 vaccines registry

Italian Ministry of Health: Serena Battilomo, Valeria Proietti.

Regional representatives: Camillo Odio (Abruzzo); Michele Recine (Basilicata); Innocenza Ruberto (Calabria); Salvatore Ascione e Massimo Bisogno (Campania); Gandolfo Miserendino, Massimiliano Navacchia (EmiliaRomagna); Beatrice Del Frate, Emanuela Cau (Friuli Venezia Giulia); Diego Baiocchi, Danilo Fusco (Lazio); Domenico Gallo (Liguria); Maria Rosa Marchetti (Lombardia); Liana Spazzafumo (Marche); Raffaele Malatesta (Molise); Antonio Fanolla (P.A. Bolzano); Diego Conforti, Carlo Trentini (P.A. Trento); Antonino Ruggeri (Piemonte); Concetta Ladalardo, Nehludoff Albano (Puglia); Marco Corona, Paolo Lombardi (Sardegna); Massimo lacono (Sicilia); Paolo Bruno Angori, Andrea Belardinelli (Toscana); Milena Solfiti (Umbria); Stefano Fioraso (Valle D’Aosta); Chiara Poma, Nadia Raccanello (Veneto).

\section{Acknowledgements}

Members of the Italian Integrated Surveillance of COVID-19 study group and the Italian COVID-19 vaccines registry are acknowledged.

\section{Conflict of interest}

None declared.

\section{Authors' contributions}

All authors fulfilled the ICJME authorship criteria. PP, FMI, $C M$ and $A M U$ designed the paper. MT, MM and MS retrieved and linked databases. SSA, MF, MP, RDC and AB carried out the analysis. AMU, AF, PP, VP, MDM, CM, FR, FDA and SB wrote the manuscripts, which was then reviewed, modified and approved by the other authors.

\section{References}

1. Agenzia Italiana del Farmaco (AIFA). Vaccini COVID-19. Rome: AIFA. [COVID-19 Vaccines]. [Accessed 19 May 2021]. Available from: https://www.aifa.gov.it/vaccini-covid-19

2. Ministero della Salute. Piano strategico per la vaccinazione anti-SARS-CoV-2/COVID-19. [Strategic plan for SARS-CoV-2 COVID-19 vaccination]. Rome: Ministero della Salute; 2020. Italian. Available from: https://www.trovanorme.salute.gov.it/ norme/renderNormsanPdf?anno $=2021 \&$ codLeg $=78657 \&$ parte $=1 \&$ serie $=$ null

3. Riccardo F, Ajelli M, Andrianou XD, Bella A, Del Manso M, Fabiani M, et al. , COVID-19 working group. Epidemiological characteristics of COVID-19 cases and estimates of the reproductive numbers 1 month into the epidemic, Italy, 28 January to 31 March 2020. Euro Surveill. 2020;25(49):2000790. . Available from: https://www.eurosurveillance.org/ content/10.2807/1560-7917.ES.2020.25.49.2000790 https:// doi.org/10.2807/1560-7917.ES.2020.25.49.2000790 PMID: 33303064

4. Polack FP, Thomas SJ, Kitchin N, Absalon J, Gurtman A, Lockhart S, et al. , C4591001 Clinical Trial Group. Safety and Efficacy of the BNT162b2 mRNA Covid-19 Vaccine. N Engl J Med. 2020;383(27):2603-15. https://doi.org/10.1056/ NEJMoa2034577 PMID: 33301246

5. Baden LR, El Sahly HM, Essink B, Kotloff K, Frey S, Novak R, et al. , COVE Study Group. Efficacy and Safety of the mRNA1273 SARS-CoV-2 Vaccine. N Engl J Med. 2021;384(5):403-16. https://doi.org/10.1056/NEJMoa2035389 PMID: 33378609

6. Voysey M, Clemens SAC, Madhi SA, Weckx LY, Folegatti PM, Aley PK, et al. , Oxford COVID Vaccine Trial Group. Safety and efficacy of the ChAdOx1 nCoV-19 vaccine (AZD1222) against SARS-CoV-2: an interim analysis of four randomised controlled trials in Brazil, South Africa, and the UK. Lancet. 2021;397(10269):99-111. https://doi.org/10.1016/S01406736(20)32661-1 PMID: 33306989 
7. Dagan N, Barda N, Kepten E, Miron O, Perchik S, Katz MA, et al. BNT162 b2 mRNA Covid-19 Vaccine in a Nationwide Mass Vaccination Setting. N Engl J Med. 2021;384(15):1412-23. https://doi.org/10.1056/NEJMoa2101765 PMID: 33626250

8. Hall VJ, Foulkes S, Saei A, Andrews N, Oguti B, Charlett A, et al. , SIREN Study Group. COVID-19 vaccine coverage in healthcare workers in England and effectiveness of BNT162 b2 mRNA vaccine against infection (SIREN): a prospective, multicentre, cohort study. Lancet. 2021;397(10286):1725-35. https://doi. org/10.1016/S0140-6736(21)00790-X PMID: 33901423

9. Haas EJ, Angulo FJ, McLaughlin JM, Anis E, Singer SR,

Khan $\mathrm{F}$, et al. Impact and effectiveness of mRNA BNT162 b2 vaccine against SARS-CoV-2 infections and COVID-19 cases, hospitalisations, and deaths following a nationwide vaccination campaign in Israel: an observational study using national surveillance data. Lancet. 2021;397(10287):181929. https://doi.org/10.1016/S0140-6736(21)00947-8 PMID: 33964222

10. Fabiani M, Ramigni M, Gobbetto V, Mateo-Urdiales A, Pezzotti $P$, Piovesan C. Effectiveness of the Comirnaty (BNT162b2, BioNTech/Pfizer) vaccine in preventing SARS-CoV-2 infection among healthcare workers, Treviso province, Veneto region, Italy, 27 December 2020 to 24 March 2021. Euro Surveill. 2021;26(17):2100420. https://doi.org/10.2807/1560-7917. ES.2021.26.17.2100420 PMID: 33928898

11. Tenforde MW, Olson SM, Self WH, Talbot HK, Lindsell CI, Steingrub JS, et al. , IVY Network, HAIVEN Investigators. Effectiveness of Pfizer-BioNTech and Moderna Vaccines Against COVID-19 Among Hospitalized Adults Aged $\geq 65$ Years - United States, January-March 2021. MMWR Morb Mortal Wkly Rep. 2021;70(18):674-9. https://doi.org/10.15585/mmwr. mm7018е1 PMID: 33956782

12. Vasileiou E, Simpson CR, Robertson C, Shi T, Kerr S, Agrawal U, et al. Effectiveness of First Dose of COVID-19 Vaccines Against Hospital Admissions in Scotland: National Prospective Cohort Study of 5.4 Million People. SSRN Electron J. 2021. [Accessed 4 May 2021]. https://doi.org/10.2139/ssrn.3789264

13. Day M. Covid-19: Stronger warnings are needed to curb socialising after vaccination, say doctors. BMJ. 2021;372(783):n783. https://doi.org/10.1136/bmj.n783 PMID: 33741565

\section{License, supplementary material and copyright}

This is an open-access article distributed under the terms of the Creative Commons Attribution (CC BY 4.0) Licence. You may share and adapt the material, but must give appropriate credit to the source, provide a link to the licence and indicate if changes were made.

Any supplementary material referenced in the article can be found in the online version.

This article is copyright of the authors or their affiliated institutions, 2021. 